\title{
BMJ Open A qualitative assessment of the impact of a uterine balloon tamponade package on decisions regarding the role of emergency hysterectomy in women with uncontrolled postpartum haemorrhage in Kenya and Senegal
}

Anna Alaska Pendleton, ${ }^{1,2}$ Abirami Natarajan, ${ }^{1,2}$ Roy Ahn, ${ }^{1,2}$ Brett D Nelson, ${ }^{1,2}$ Melody J Eckardt, ${ }^{1}$ Thomas F Burke $^{1,2}$

To cite: Pendleton AA, Natarajan A, Ahn R, et al. A qualitative assessment of the impact of a uterine balloon tamponade package on decisions regarding the role of emergency hysterectomy in women with uncontrolled postpartum haemorrhage in Kenya and Senegal. BMJ Open 2016;6:e010083. doi:10.1136/bmjopen-2015010083

- Prepublication history and additional material is available. To view please visit the journal (http://dx.doi.org/ 10.1136/bmjopen-2015010083).

Received 23 September 2015 Accepted 10 December 2015

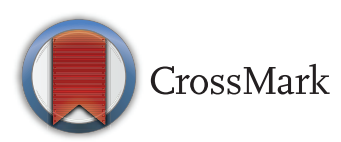

${ }^{1}$ Division of Global Health and Human Rights, Department of Emergency Medicine, Massachusetts General Hospital, Boston, Massachusetts, USA

${ }^{2}$ Harvard Medical School, Boston, Massachusetts, USA

Correspondence to Dr Thomas F Burke; tfburke@partners.org

\section{ABSTRACT}

Objectives: To assess the impact of a every second matters for mothers and babies uterine balloon tamponade package (ESM-UBT) on provider decisions regarding emergency hysterectomy in cases of uncontrolled postpartum haemorrhage (PPH).

Design: Qualitative assessment and analysis of a subgroup extracted from a larger database that contains all UBT device uses among ESM-UBT trained health providers.

Setting: Health facilities in Kenya and Senegal with ESM-UBT training and capable of performing emergency hysterectomies.

Participants: All medical doctors who had placed a UBT for uncontrolled PPH subsequent to implementation of ESM-UBT at their facility, and who also had the capabilities of performing emergency hysterectomies.

Primary outcome measures: The impact of ESMUBT on decisions regarding emergency hysterectomy in cases of uncontrolled PPH.

Results: 30 of the 31 medical doctors (97\%) who fulfilled the inclusion criteria were independently interviewed. Collectively the interviewed medical doctors had placed over 80 UBT devices for uncontrolled PPH since ESM-UBT implementation. All 30 responded that UBT devices immediately controlled haemorrhage and prevented women from being taken to emergency hysterectomy. All 30 would continue to use UBT devices in future cases of uncontrolled PPH.

Conclusions: These preliminary data suggest that following ESM-UBT implementation, emergency hysterectomy for uncontrolled PPH may be averted by use of uterine balloon tamponade.

\section{INTRODUCTION}

Postpartum haemorrhage (PPH) is the leading cause of maternal mortality globally and is responsible for greater than one in
Strengths and limitations of this study

A key strength of this study was the emergence of robust thematic consensus from all 30 independently interviewed providers that uterine balloon tamponade package (UBT) averted hysterectomy, saved the lives of women suffering from postpartum haemorrhage (PPH), and would be continued to be used in future cases of PPH.

- Social desirability and recall bias were potential weaknesses, however, efforts were made to prevent and mitigate this bias by encouraging providers to describe the strengths and challenges of every second matters for mothers and babies-UBT and by assuring all providers that answers would be de-identified.

- Although interviews were conducted with 30 providers from 13 different healthcare facilities in two countries, practices and perceptions of these providers may not necessarily reflect those of providers in other resource-limited settings.

three maternal deaths in sub-Saharan Africa. ${ }^{12}$ The fifth Millennium Development Goal sought to reduce the maternal mortality ratio by $75 \%$, from 1990 to 2015 , but many countries did not achieve this goal. ${ }^{3}$ Thirty-four per cent of maternal deaths in Kenya and Senegal remain attributable to PPH. $^{2} 3$

In the event of severe PPH refractory to uterotonic medications and manoeuvers such as uterine massage and bimanual compression, emergency hysterectomy and other surgical interventions (eg, compression sutures) are potential second-line options to address uncontrolled haemorrhage. ${ }^{4}$ However, it has 
been well described that access to surgery is severely limited across resource-constrained settings. ${ }^{5-7}$ Although uterine balloon tamponade (UBT) was introduced by the WHO in 2012 as a potential rescue intervention for severe uncontrolled PPH refractory to uterotonics, training programmes and UBT devices have not yet been integrated into health systems at scale. ${ }^{45}$

The authors previously designed and have been implementing through local partners a condom-balloon UBT kit and training package for uncontrolled PPH called Every Second Matters for Mothers and Babies UBT (ESM-UBT). Consisting of a syringe, a Luer lock valve, and a condom fastened to the end of a Foley catheter with cotton string, the ESM-UBT device may be inflated with clean water via a $60 \mathrm{~mL}$ syringe and inserted into the uterine cavity of a woman suffering from PPH. The ESM-UBT package includes a $3 \mathrm{~h}$ PPH management training, checklist wall charts, and a set of UBT devices. As of 15 September 2015, ESM-UBT had been implemented in eight resource-limited countries over the preceding 3 years. Preliminary findings on ESM-UBT demonstrate that $98 \%$ of women with severe uncontrolled PPH survive if delivery occurs at an ESM-UBT trained facility. 89

During ESM-UBT training, providers were taught that the uterine balloon was to be placed as a rescue device when PPH was refractory to uterotonics and other supportive measures. Although prior studies have demonstrated that UBT may be effectively employed in cases of severe PPH to avert maternal mortality, no prior studies have examined the impact of UBT on provider decisions regarding surgical management of $\mathrm{PPH}^{8}{ }^{9}$ This study specifically sought to assess the impact of introduction of the ESM-UBT package on provider decisions regarding emergency hysterectomy in cases of uncontrolled $\mathrm{PPH}$.

\section{SUBJECTS AND METHODS}

This study was conducted as a subset of an ongoing multinational research project examining the safety and efficacy of the ESM-UBT package to address uncontrolled PPH in resource-limited settings. The subjects for this study were medical doctors, identified from the Kenya and Senegal ESM-UBT database, who met the following criteria: (1) the provider had received ESM-UBT training between August 2012 and February 2015, (2) the provider had implemented UBT in one or more women with uncontrolled PPH since training, and (3) the provider had the capability of performing an emergency hysterectomy for PPH at the facility at which he or she inserted UBT device(s). All doctors who met these criteria were sought for interview.

Interviews were conducted between 19 January 2015 and 5 February 2015. A local translator was utilised when necessary. Interviews were performed in a semistructured format following a 24-question interview guide developed specifically for the purposes of this study (see online supplementary file). All responses were recorded, transcribed and independently analysed and coded by two researchers using NVivo V.10 software (QSR International, Doncaster, Victoria, Australia). Researchers used an iterative process of code identification and revision to develop domains and themes. ${ }^{10}$ Ethical approval was obtained from the Partners Healthcare Human Research Committee (Boston, Massachusetts, USA) and the Maseno University Ethics Review Committee (Maseno, Kenya). Informed consent was obtained prior to interviews.

\section{RESULTS}

Thirty-one medical doctors met all three inclusion criteria, and 30 were available for interview. One doctor was untraceable, having previously provided maternal health services in Garissa County, Kenya.

At the time of their interviews, 13 providers $(43 \%)$ were general medical officers, $8(27 \%)$ obstetrician/ gynaecologists $(\mathrm{Ob} / \mathrm{Gyn}), 6(20 \%) \mathrm{Ob} / \mathrm{Gyn}$ residents, $2(7 \%)$ surgical residents, and 1 a general surgeon. The 30 providers had a median of 5.5 years of clinical experience since graduating from medical school (range $1-46$ years). Twenty-eight of the 30 interviewed providers $(93 \%)$ reported caring for at least one (15 had more than five each) maternal death secondary to uncontrolled PPH during their careers. The majority of providers $(18$ of $30,60 \%)$ reported that they had received no instruction on UBT during their medical education (table 1).

Interviewed providers collectively placed over 80 UBT devices for uncontrolled PPH at seven facilities in Senegal and at six facilities in Kenya between January 2012 and January 2015 (table 1). Nine providers (30\%) reported having inserted a UBT device in a single case of uncontrolled $\mathrm{PPH}$, while $21(70 \%)$ reported inserting multiple UBT devices. Only one provider reported a case of maternal death subsequent to insertion of a UBT device. The provider reported that although insertion of the UBT device resulted in cessation of bleeding from the uterus, return of consciousness, and improvement in the patient's blood pressure, the mother subsequently developed disseminated intravascular coagulation and died in transit to a referral facility. No other cases of PPH-associated maternal death subsequent to UBT insertion were reported.

Twenty-six of the 30 providers $(87 \%)$ responded that had they not received UBT training, they would have performed emergency hysterectomies in each of the cases of uncontrolled PPH that they instead successfully managed with UBT devices. Eight providers (27\%) suggested that they might have tried to perform an intermediate procedure such as B-Lynch suture prior to hysterectomy. Alternate courses suggested by the four providers who did not propose immediate hysterectomy as a treatment modality in the absence of ESM-UBT included exam under anaesthesia and consultation with 
an $\mathrm{Ob} / \mathrm{Gyn}$. One provider responded that without UBT training, "We would have lost many mothers even before referral to be honest." All 30 providers responded that they believed UBT to be "successful in controlling the bleeding from uncontrolled PPH." One provider responded that while she believed that UBT "helped control the bleeding," expulsion of the placental tissues was also necessary for bleeding cessation in the one case that she had implemented UBT. All of the providers responded that UBT prevented women with severe uncontrolled PPH from being sent to surgery, and all 30 reported that they would use UBT devices in future cases of uncontrolled PPH.

Several major themes emerged from the provider interviews as rationale for UBT implementation (box 1). Fifteen providers $(50 \%)$ mentioned ease of use as an advantage of UBT as compared to surgery, eight

\begin{tabular}{ll} 
Table $1 \quad$ Interviewed provider training & \\
\hline Level of training & $\mathbf{N}=\mathbf{3 0}$ \\
\hline General medical officer & $13(43 \%)$ \\
Ob/Gyn consultant & $8(27 \%)$ \\
Ob/Gyn resident & $6(20 \%)$ \\
General surgery resident & $2(7 \%)$ \\
General Surgeon consultant & $1(3 \%)$ \\
\hline Years of clinical experience since & $\mathbf{N}=\mathbf{3 0}$ \\
graduation from medical school & \\
\hline$<5$ & $10(33 \%)$ \\
$5-10$ & $14(47 \%)$ \\
$>10$ & $6(20 \%)$ \\
\hline UBT training during medical school & $\mathbf{N}=\mathbf{3 0}$ \\
\hline No UBT training & $18(60 \%)$ \\
Mention of UBT in text or lecture without & $9(30 \%)$ \\
practical training & $1(3 \%)$ \\
UBT training in medical school & $2(7 \%)$ \\
\hline Unable to recall & $\mathbf{N}=\mathbf{3 0}$ \\
\hline Estimated cases of uncontrolled PPH in & \\
the prior year & $3(10 \%)$ \\
\hline$<5$ cases & $5(17 \%)$ \\
$5-10$ cases & $22(73 \%)$ \\
\hline$>10$ cases or 'many' & $\mathbf{N}=\mathbf{2 8}$ \\
\hline Estimated cases of mortality secondary to & \\
\hline PPH in career & $13(46 \%)$ \\
\hline$<5$ cases & $6(21 \%)$ \\
$5-10$ cases & $9(32 \%)$ \\
\hline$>10$ cases or 'many' & $\mathbf{N}=\mathbf{3 0}$ \\
\hline Total number of cases of PPH treated with & \\
\hline UBT & $9(30 \%)$ \\
\hline 1 UBT cases & $11(37 \%)$ \\
\hline-5 UBT cases & $10(33 \%)$ \\
\hline Ob/Gyn, obstetrician/gynaecologists; PPH, postpartum \\
hemorrhage; UBT, uterine balloon tamponade package. \\
\hline
\end{tabular}

providers $(27 \%)$ mentioned reduced cost, and six providers $(20 \%)$ mentioned reduced time required for UBT as compared to surgery. Reduced invasiveness was cited by four providers $(13 \%)$, and greater efficacy was cited by $14(47 \%)$ as advantages of UBT. Seven providers (23\%) cited practical difficulties performing surgery, such as obtaining blood transfusions or operating room space, as motivation for UBT. Of the 30 providers, 10 (33\%) mentioned a psychosocial advantage of UBT compared to surgical intervention. These providers discussed the emotional and social impact of hysterectomy on women of childbearing age, especially in the cases of women from regions where loss of one's uterus is considered equivalent to loss of womanhood.

\section{DISCUSSION}

PPH is the leading cause of maternal death and disability worldwide and remains at crisis levels in resourcelimited settings. Although surgeries such as hysterectomy may be used to address uncontrolled PPH refractory to first-line therapies, surgical intervention is often unavailable and may additionally be undesirable due to financial burden, operative risks and psychosocial sequelae.

While ESM-UBT has recently been shown to be remarkably effective in arresting haemorrhage and saving lives in women with uncontrolled PPH, UBT training is rare and UBT device availability is scarce. ${ }^{8}$ This study sought to understand whether ESM-UBT could additionally contribute to averting emergency hysterectomies for uncontrolled PPH. The 30 interviewed providers collectively had considerable clinical experience, and most had significant experience treating severe $\mathrm{PPH}(73 \%$ had treated more than 10 cases of severe PPH in the prior year alone). All described UBT as successful in controlling PPH-related haemorrhage and preventing emergency hysterectomies. Each of the 30 providers reported that he or she would use UBT in future cases of uncontrolled PPH.

Providers described that their rational for using UBT included ease of use, improved efficiency and decreased time to PPH control as opposed to surgical interventions. In addition, the benefit for women included decreased risk through avoiding surgical interventions in potentially severely anaemic states, and avoiding the psychosocial consequences of loss of uterus and fertility. In this way, UBT may avert the significant physical, emotional and psychosocial morbidity associated with emergency hysterectomy.

In summary, this study demonstrates that following a short, structured UBT training course, medical personnel with diverse clinical experience and operating at a wide range of facilities across two countries could easily and effectively apply UBT. Although prior literature suggests that UBT may be used to arrest haemorrhage and save lives of women suffering from uncontrolled PPH, this study provides first-ever evidence that UBT may also be used to avert emergency hysterectomy in cases of 
Box 1 Major themes and demonstrative subject quotations regarding uterine balloon tamponade package (UBT) use

Provider experience with UBT in management of uncontrolled postpartum haemorrhage (PPH)

- UBT resulted in cessation of haemorrhage within a few minutes of insertion for the majority of providers.

"In all of my three cases, they were all success stories. I put in my UBT and then like magic I observed... in the next 10 min, she was completely dry. No bleeding at all."-obstetrician/gynaecologists (Ob/Gyn) resident

- Insertion of UBT resulted in observable improvement of patient clinical status such as increase in blood pressure or return of consciousness.

"Most of the time if you put it when there are already signs of hypotension/confusion, all that improves with time. Usually within 30 min your patient is stable."-Surgery resident

"Before the UBT the patient was unconscious with low blood pressure (BP). Then after, the BP is going up and she became conscious."-Ob/Gyn resident

- UBT prevented women on their way to or already within the operating room from undergoing hysterectomy for control of severe $\mathrm{PPH}$.

"For a surgeon we cut. I tell you, when I went there, my thing was, "Blood would not cost much, let's take her to theatre." Then the nurse told me, "Why don't you do UBT?" And I said, "Oh, yes, bring it." Actually for me it would have just been hysterectomy, subtotal or whichever would apply."-General surgeon

"Surgery was the first option on my mind, because even the patient had already been wheeled to theatre. The next step was I go in for surgery."-Ob/Gyn resident

- UBT was effectively employed to treat PPH in cases in which the provider was precluded from performing surgery by patient or facility factors.

"The patient had bled a lot-actually going into hypovolemic shock. Patient paper white with a very low[hemoglobin], and then we didn't have blood at all. So here I am with my anaesthetic team. We have to do something to control this bleeding. We have to go in. But they're like, "We're not sure if this patient can withstand the anaesthetic drug." So I'm like, what can I do? The patient can't be referred from Dadaab to Nairobi. We've called all of the neighbouring hospitals, there's no blood. So my next question was, am I going to watch a patient die in my hands when there's nothing I can do at all? So in the course of trying to see what I can do for the patient, I remembered the UBT... I immediately did UBT."-Ob/Gyn resident

- The majority of providers would have performed hysterectomy for the case(s) in which UBT was used if they had received no prior UBT training.

"We'd need to go to theatre. And l've gone to the theatre either for the B-lynch or hysterectomy or cauterisation. But because of the balloon tamponade and the success that it has, there were mothers who didn't have to go to theatre."-Medical officer

- All providers advocated that UBT prevented women with uncontrolled PPH from undergoing surgery.

Interviewer: "Do you think it prevented her from having to have surgery then?

Participant: "Yes. It prevented her. Because I was actually itching to cut her and it prevented it... So it still prevented her from having surgery. And you will imagine, then having other pregnancies."-General surgeon
- All providers plan to use UBT in future cases of uncontrolled $\mathrm{PPH}$.

"I will always use. If I've tried everything and it's failed, before thinking of surgery, I will always try a UBT."-Medical officer

\section{UBT advantages}

- Providers perceived efficacy and ease of use as the two greatest advantages of UBT and the two factors most supporting future device implementation

"I feel that from my experience that I have had, the UBT is effective in controlling bleeding. And much more even, in the resourcelimited setups where I used to work, where you don't have all the things that you need to do all of the complex procedures, a simple thing like the UBT kit done effectively would ensure that you save a life."-Medical officer

"The strengths of the UBT are: 1) the simplicity. It is a simple kit. It's a simple procedure. The other things is that it's something that can be trained to all levels of staff. It can be trained to the doctors, the nurses, the clinical officers, even the nurse aides. It is something which can be trained to any health worker proficient with medical training... I think it is a big step that something that is very simple to construct, easy to procure, and the results are tremendous."-Ob/Gyn resident

- Reduced time, cost, and invasiveness also factored strongly in providers' decisions to implement UBT.

"The first thing is finance-the low cost as compared to hysterectomy.[You] don't need to give drugs, like surgery you need anesthesia and so and so. You can go faster compared to surgery."Ob/Gyn resident

- UBT represents an intervention that can be effectively implemented in limited-resource facilities where surgical support staff, blood, operating room space and equipment are unavailable.

"We have the resource-poor centres and you have a whole country with one gynaecologist, so sometimes medical officers do hysterectomies. So UBT is one of the first things actually we like to use."-Medical officer

- Besides averting the physical and psychosocial consequences of emergency hysterectomy, UBT also represents a life-saving intervention to address PPH in regions where hysterectomy is not permitted by social mores.

"First of all, these were women who were still the childbearing age... and then the community we were in, they were not so open to surgery or anything like that... Even the caesarean section, it's usually very hard just convincing them this is a caesarean section that has to be done... So the community we were in, surgery was never an option."-Medical officer

uncontrolled PPH. ${ }^{11}$ Further work is needed to help integrate UBT training in medical curricula, as well as in continued education programmes for medical providers. Additional studies are needed to quantify the impact of ESM-UBT on rates of emergency surgery for uncontrolled PPH, evaluate differences in outcomes between UBT and emergency surgery, understand optimal ESM-UBT application and devise successful expansion and sustainability strategies.

Acknowledgements The authors thank Drs Khadija Abdalla and Mansour Niang for their support in gaining access to the interviewed providers. The authors additionally thank Geoff Karanja and Emily DeRedon for their tireless assistance with complex logistics. 
Contributors AAP and AN led study design, data collection and participated in manuscript writing; RA, BDN, and MJE supported study design and data collection; and TFB conceived of the study, supported study design and data collection and led manuscript preparation.

Funding The Every Second Matters for Mothers and Babies Uterine Balloon Tamponade research program (ESM-UBT) is made possible through the generous support of the Izumi Foundation, the Ujenzi Charitable Trust, Harvard Medical School and the Saving Lives at Birth partners: the USA Agency for International Development (USAID), the Government of Norway, the Bill \& Melinda Gates Foundation, Grand Challenges Canada, and the UK Government. This manuscript was prepared by the authors and does not necessarily reflect the views of the funding partners.

Competing interests None declared.

Ethics approval Partners Healthcare Human Research Committee (Boston, Massachusetts, USA) and the Maseno University Ethics Review Committee (Maseno, Kenya).

Provenance and peer review Not commissioned; externally peer reviewed.

Data sharing statement No additional data are available.

Open Access This is an Open Access article distributed in accordance with the Creative Commons Attribution Non Commercial (CC BY-NC 4.0) license, which permits others to distribute, remix, adapt, build upon this work noncommercially, and license their derivative works on different terms, provided the original work is properly cited and the use is non-commercial. See: http:// creativecommons.org/licenses/by-nc/4.0/

\section{REFERENCES}

1. World Health Organization, UNICEF, UNFPA, The World Bank. Trends in maternal mortality: 1990 to 2008. http://whqlibdoc.who.int/ publications/2010/9789241500265_ eng.pdf Published 2010 (accessed 24 Apr 2015).

2. Khan KS, Wojdyla D, Say L, et al. WHO analysis of causes of maternal death: a systematic review. Lancet 2006;367:1066-74.

3. World Health Organization, UNICEF, UNFPA, The World Bank and the United Nations Population Division. Trends in maternal mortality: 1990 to 2013. http://apps.who.int/iris/bitstream/10665/112682/2/ 9789241507226_eng.pdf?ua=1 Published 2014 (accessed Apr 2015).

4. WHO. Recommendations for the management and treatment of postpartum hemorrhage and retained placenta. Geneva: World Health Organization, 2012.

5. Tindell K, Garfinkel R, Abu-Haydar E, et al. Uterine balloon tamponade for the treatment of postpartum haemorrhage in resource-poor settings: a systematic review. BJOG 2013;120:5-14.

6. Doumouchtsis SK, Papageorghiou AT, Arulkumaran S. Systematic review of conservative management of postpartum hemorrhage: what to do when medical treatment fails. Obstet Gynecol Surv 2007;62:540-7.

7. Elmir R, Schmied V, Jackson D, et al. Between life and death: women's experiences of coming close to death, and surviving a severe postpartum haemorrhage and emergency hysterectomy. Midwifery 2012;28:228-35.

8. Burke TF, Ahn R, Nelson B, et al. A postpartum hemorrhage package with condom uterine balloon tamponade: a prospective multi-center case series in Kenya, Sierra Leone, Senegal, and Nepal. BJOG 2015; in press.

9. Nelson BD, Stoklosa H, Ahn R, et al. Uterine balloon tamponade for postpartum hemorrhage control among community-based health providers in South Sudan. Intl J Gynecology Obstetrics 2013:122:27-32.

10. Bradley EH, Curry LA, Devers KJ. Qualitative data analysis for health services research: developing taxonomy, themes, and theory. Health Services Research 2007;42:1758-72.

11. Georgiou C. Balloon tamponade in the management of postpartum haemorrhage: a review. BJOG 2009;116:748-57. 